\title{
Micellization Behavior of an Amphiphilic Statistical Copolymer in Water-Methanol Mixtures
}

\author{
By Takuya MoRI ${ }^{\dagger}$ Akihito HASHIDZUME, and Takahiro SATO*
}

Sedimentation equilibrium measurements were made on an amphiphilic statistical copolymer p(AMPS/C6) made of sodium 2-(acrylamido)-2-methylpropane-sulfonate (AMPS) and $n$-hexyl methacrylate (C6), dissolved in water-methanol mixtures with $0.2 \mathrm{M} \mathrm{LiClO}_{4}$ and different methanol contents over a wide polymer concentration range. The experimental results were analyzed in terms of a micellar solution theory for amphiphilic polyelectrolytes to estimate the aggregation number $m$ of the micelle and the internal free energy change $\Delta_{m} / m$ (per polymer chain) due to micellization. Both $m$ and $\Delta_{m} / m$ decreased with increasing the methanol content in the solvent. In addition, dynamic light scattering for the same copolymer system indicated that $\mathrm{p}(\mathrm{AMPS} / \mathrm{C} 6)$ formed a uni-core star-like micelle in water-methanol mixture with the methanol mole fractions of 0 and 0.06 .

KEY WORDS: Micelle / Amphiphilic Polymer / Statistical Copolymer / Sedimentation Equilibrium / Dynamic Light Scattering /

Amphiphilic copolymers form micelles in aqueous media due to the strong hydrophobic interaction among the polymer chains. ${ }^{1}$ The micellization is important for their applications to the rheology modifier, emulsifier, drag delivery system, and so on, ${ }^{2-4}$ because the micellization changes remarkably their solution properties. In the practical applications for those purposes, their aqueous solutions are often mixed with various additives, e.g., low molar mass organic compounds, salts, surfactants, and other polymers. Those additives can modify the micellization behavior of the polymers in aqueous media by interacting with the hydrophobic or hydrophilic moieties of the copolymer chain, so that it is essentially important to understand the effect of additives on the micellization behavior in aqueous solutions of amphiphilic copolymers.

Recently, Hashidzume et al. ${ }^{5}$ investigated the micellar structure of an amphiphilic statistical copolymer $\mathrm{p}$ (AMPS/C6), made of sodium 2-(acrylamido)-2-methylpropane-sulfonate (AMPS) and $n$-hexyl methacrylate (C6), in aqueous salt solution. The copolymer aggregation number $m$ of the micelle and the number of hydrophobic cores per micelle depended on the copolymer molecular weight and hydrophobic monomer content. Furthermore, their hydrodynamic radius data indicated that the copolymer formed a uni-core star-like micelle at low copolymer molecular weights.

Afterward, Nojima et al. ${ }^{6}$ refined the data analysis for the $\mathrm{p}$ (AMPS/C6) micelle in aqueous salt solution by taking into account the association-dissociation equilibrium in the micellar solution. Using a micellar solution theory for amphiphilic polyelectrolytes, they estimated more precise $m$ for the $\mathrm{p}$ (AMPS/C6) micelle in aqueous salt solution.

In the present study, we have made sedimentation equilibrium and dynamic light scattering measurements on $\mathrm{p}$ (AMPS/C6) dissolved in water-methanol mixtures with $0.2 \mathrm{M} \mathrm{LiClO}_{4}$ and different methanol mole fraction $x_{\text {methanol }}$. Applying the micellar solution theory, we have estimated $m$ and also the internal free energy change $\Delta_{m} / m$ due to micellization as functions of the methanol content, from the sedimentation equilibrium data. On the other hand, we have analyzed the dynamic light scattering results to elucidate the micellar structure in aqueous and aqueous methanol solutions. It was found that the micellization behavior of $\mathrm{p}$ (AMPS/C6) was very sensitive to the addition of small amount of methanol, which weakened the hydrophobic interaction among hexyl groups on copolymer chains.

\section{EXPERIMENTAL}

\section{p(AMPS/C6) Sample and Test Solutions}

A statistical copolymer sample of p(AMPS/C6) was obtained by the reversible addition-fragmentation chain transfer (RAFT) polymerization, ${ }^{7-9}$ as reported previously. ${ }^{5}$ The sample was purified by reprecipitation and dialysis, and then freeze-dried from aqueous solution.

The weight-average molecular weight $M_{\mathrm{w}}$, the ratio $M_{\mathrm{z}} / M_{\mathrm{w}}$ of the z-average molecular weight to $M_{\mathrm{w}}$, and the second virial coefficient $A_{2}$ of the copolymer sample obtained were determined in methanol with $0.2 \mathrm{M} \mathrm{\textrm {LiClO } _ { 4 }}$ at $25^{\circ} \mathrm{C}$ by sedimentation equilibrium ( $c f$. below). The mole fraction $x$ of the hydrophobic monomer units in the copolymer sample was estimated by ${ }^{1} \mathrm{H}$ NMR using $\mathrm{D}_{2} \mathrm{O}$ as the solvent at $30{ }^{\circ} \mathrm{C} .{ }^{5} \mathrm{All}$ the results are listed in Table I. The $M_{\mathrm{z}} / M_{\mathrm{w}}$ value indicates a narrow molecular weight distribution of the sample prepared by the RAFT polymerization.

Water, methanol, and their mixtures with different compositions all of which contained $0.2 \mathrm{M} \mathrm{LiCliO}_{4}$ were used as solvents. The freeze-dry sample of p(AMPS/C6) was directly dissolved in those solvents. In what follows, the solvent composition is expressed in terms of the mole fraction $x_{\text {methanol }}$ of methanol in the solvent.

\footnotetext{
Department of Macromolecular Science, Osaka University, 1-1 Machikaneyama-cho, Toyonaka 560-0043, Japan

Present Address: Sumitomo Chemical Co. Ltd., 1-98, Kasugadenaka 3-chome, Konohana-ku, Osaka 554-8558, Japan

*To whom correspondence should be addressed (Tel: +81-6-6850-5461, Fax: +81-6-6850-5461, E-mail: tsato@ chem.sci.osaka-u.ac.jp).
} 
Table I. Molecular characteristics of copolymers in methanol with $0.2 \mathrm{M} \mathrm{LiClO}_{4}$

\begin{tabular}{ccccc}
\hline sample & $\left.M_{\mathrm{w}} / 10^{4} \mathrm{a}\right)$ & $M_{\mathrm{z}} / M_{\mathrm{w}}$ a) & $x^{\mathrm{b})}$ & $A_{2} / 10^{-3} \mathrm{~mol} \mathrm{~cm}^{3} \mathrm{~g}^{-2}$ a) \\
\hline $\mathrm{p}$ (AMPS/C6) & 1.69 & 1.17 & 0.15 & 1.00 \\
\hline
\end{tabular}

The above data were determined by a) sedimentation equilibrium or b) ${ }^{1} \mathrm{H}$ NMR

\section{Measurements}

Sedimentation equilibrium measurements on $\mathrm{p}$ (AMPS/C6) solutions with different $x_{\text {methanol }}$ were performed at $25.0^{\circ} \mathrm{C}$ using an Optima XL-I type ultracentrifuge (Beckman-Coulter) equipped with a Rayleigh interference optical system with a $675 \mathrm{~nm}$ light emitting from a diode laser. The concentration distribution in the solution under the centrifugal field was determined by the interferometry using the specific refractive index increment $(\partial n / \partial c)_{\mu}$ at constant solvent chemical potential.

The apparent molecular weight $M_{\text {app }}$ was calculated from $^{10,11}$

$$
M_{\text {app }}^{-1}=\frac{\omega^{2}\left(r_{\mathrm{b}}^{2}-r_{\mathrm{a}}^{2}\right) c_{0}(\partial \rho / \partial c)_{\mu}}{2 R T\left(c_{\mathrm{b}}-c_{\mathrm{a}}\right)}
$$

where $r_{\mathrm{b}}$ and $r_{\mathrm{a}}$ are the distance from the center of revolution to the cell bottom and meniscus, respectively, $c_{\mathrm{b}}$ and $c_{\mathrm{a}}$ are polymer mass concentrations at $r_{\mathrm{b}}$ and $r_{\mathrm{a}}$ respectively, $\omega$ is the angular velocity, $c_{0}$ is concentration of solution before centrifugation, and $(\partial \rho / \partial c)_{\mu}$ is the specific density increment at constant solvent chemical potential; $R T$ is the gas constant multiplied by the absolute temperature. In dilute methanol solutions where $\mathrm{p}$ (AMPS/C6) is molecularly dispersed, ${ }^{5}$ the equation $M_{\mathrm{app}}{ }^{-1}=1 / M_{\mathrm{w}}+2 A_{2} \bar{c}$ holds where $\bar{c}=\left(c_{\mathrm{a}}+c_{\mathrm{b}}\right) / 2$, and we can estimate $M_{\mathrm{w}}$ and $A_{2} \cdot{ }^{12}$ Using the established procedure, we can also estimate $M_{\mathrm{z}} / M_{\mathrm{w}}$ from sedimentation equilibrium data for dilute solutions. ${ }^{13}$

Values of $(\partial n / \partial c)_{\mu}$ and $(\partial \rho / \partial c)_{\mu}$ of $\mathrm{p}(\mathrm{AMPS} / \mathrm{C} 6)$ at different $x_{\text {methanol }}$ were measured using solutions dialyzed against their solvents at $25^{\circ} \mathrm{C}$ for a week. A modified SchulzCantow type differential refractometer (Shimadzu) and a DMA5000 densitometer (Anton Paar) were used for refractometry and densitometry. The results summarize in Table II. The partial specific volume $\bar{v}$ of $\mathrm{p}$ (AMPS/C6) at each $x_{\text {methanol, }}$, calculated from $\bar{v}=\left[1-(\partial \rho / \partial c)_{\mu}\right] / \rho_{0}\left(\rho_{0}\right.$ : the solvent density) is also listed in Table II.

Dynamic light scattering measurements for copolymer solutions at $x_{\text {methanol }}=0$ and 0.06 were performed using an ALV/DLS/SLS-5000 light scattering system equipped by an ALV-5000 multiple $\tau$ digital correlator at $25^{\circ} \mathrm{C}$. Vertically polarized light with the wavelength $\lambda_{0}$ of $532 \mathrm{~nm}$ emitted from

Table II. Values of $(\partial n / \partial c)_{\mu}$ and $(\partial \rho / \partial c)_{\mu}$ for $\mathrm{p}(\mathrm{AMPS} / \mathrm{C} 6)$ in methanol-water mixtures at $25^{\circ} \mathrm{C}$

\begin{tabular}{cccccc}
\hline$x_{\text {methanol }}$ & 0 & 0.06 & 0.13 & 0.31 & 1 \\
\hline$\partial n / \partial c(675 \mathrm{~nm}) / \mathrm{g}^{-1} \mathrm{~cm}^{3}$ & 0.128 & 0.142 & 0.147 & 0.146 & 0.146 \\
$\partial \rho / \partial c$ & 0.211 & 0.320 & 0.334 & 0.403 & 0.599 \\
$\overline{\boldsymbol{v}} / \mathrm{cm}^{3} \mathrm{~g}^{-1}$ & 0.782 & 0.684 & 0.682 & 0.637 & 0.499 \\
\hline
\end{tabular}
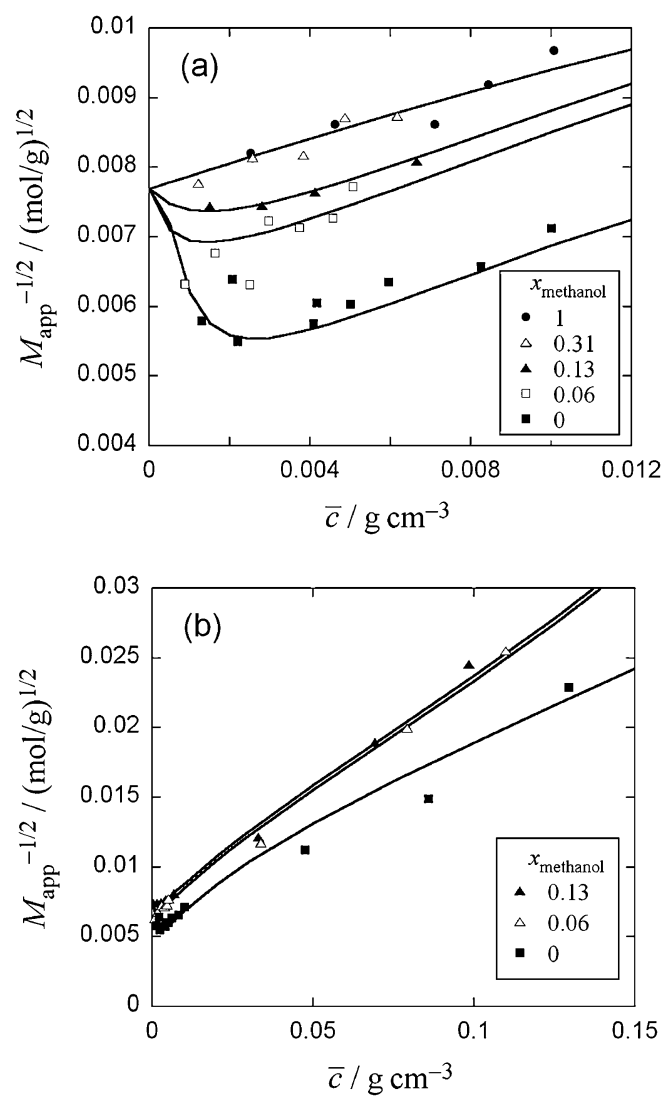

Figure 1. Concentration dependence of the apparent molecular weight $M_{\mathrm{app}}$ obtained by sedimentation equilibrium for the amphiphilic statistical copolymer $\mathrm{p}(\mathrm{AMPS} / \mathrm{C} 6)$ dissolved in water-methanol mixtures with $0.2 \mathrm{M} \mathrm{LiClO}_{4}$ and different methanol mole fraction $x_{\text {methanol }}$. (a) Data in a dilute region; (b) data in a wide concentration range; Curves: the micellar solution theory for amphiphilic polyelectrolytes (see the text) to determine the aggregation number $m$ and the internal free energy change $\Delta_{m} / m$ due to the micellization.

a Nd:YAG laser was used as an incident light, and the scattered light was measured with no analyzer. Test solutions for light scattering measurements were optically purified by filtration through a $0.8 \mu \mathrm{m}$ membrane filter, followed by a $3 \mathrm{~h}$ centrifugation at $9000 \mathrm{rpm}$ prior to measurements. The intensity autocorrelation function obtained by dynamic light scattering was analyzed by a CONTIN program to estimate the spectrum $A(\tau, k)$ of the relaxation time $\tau$ in the logarithmic scale at each scattering angle or the magnitude of the scattering vector $k$.

\section{RESULTS}

Figure 1 shows plots of $M_{\text {app }}{ }^{-1 / 2}$ vs. $\bar{c}$ obtained by sedimentation equilibrium for $\mathrm{p}$ (AMPS/C6) in water-methanol mixtures with $0.2 \mathrm{M} \mathrm{LiClO}_{4}$. In Panel a showing a dilute region, data points at $x_{\text {methanol }}=1$ (filled circles) almost follow the straight line, from which we have determined $M_{\mathrm{w}}$ and $A_{2}$, already shown in Table I. With decreasing $x_{\text {methanol }}$, data points shift downward at $x_{\text {methanol }}<0.31$, indicating the micellization of $\mathrm{p}$ (AMPS/C6) chains. Although data points at $x_{\text {methanol }}=0$ (filled squares) slightly scatter, they seem to obey a curve 

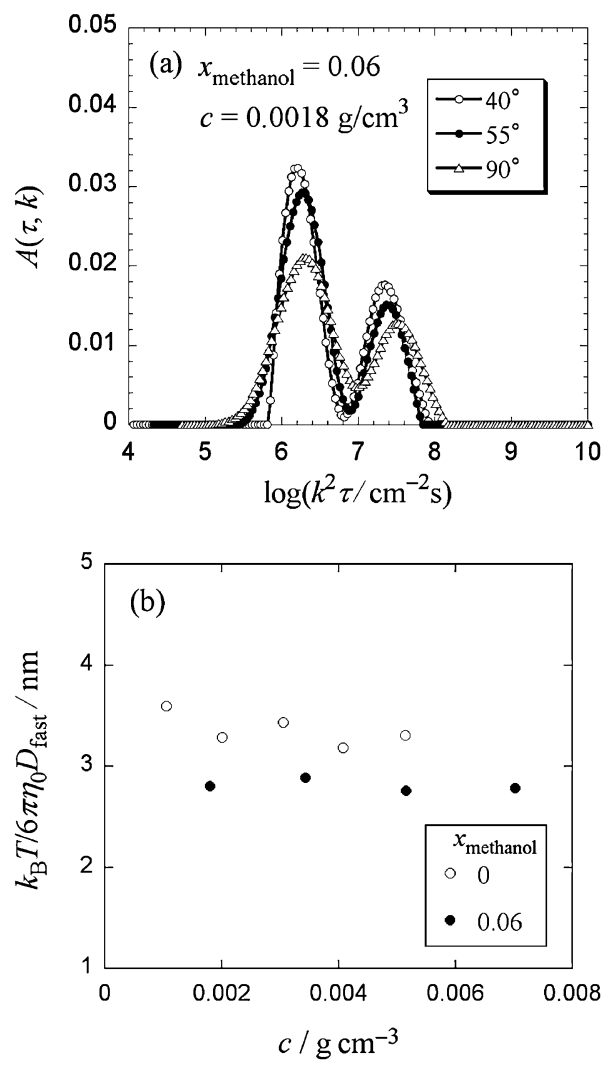

Figure 2. (a) Relaxation time spectra $A(\tau, k)$ obtained by dynamic light scattering for a $\mathrm{p}$ (AMPS/C6) solution with $x_{\text {methanol }}=0.06$ and $c=0.0018 \mathrm{~g} / \mathrm{cm}^{3}$ at three different scattering angles; (b) Concentration dependence of the diffusion coefficient (of the fast relaxation component) for $\mathrm{p}(\mathrm{AMPS} / \mathrm{C} 6)$ dissolved in water-methanol mixtures with $0.2 \mathrm{M} \mathrm{LiClO}_{4}$ at $x_{\text {methanol }}=0$ and 0.06 .

convex downward. In Panel $\mathrm{b}$ of a wider concentration range, $M_{\text {app }}{ }^{-1 / 2}$ monotonically increases with $\bar{c}$. Even at $\bar{c}>0.1 \mathrm{~g} /$ $\mathrm{cm}^{3}$, solution viscosities were still low and gelation was not observed for all solutions examined.

Figure 2a illustrates examples of the relaxation time spectra $A(\tau, k)$ obtained by dynamic light scattering ( $x_{\text {methanol }}=0.06$ and $c=0.018 \mathrm{~g} / \mathrm{cm}^{3}$ ). The spectra are bimodal, being similar to those reported previously for other $\mathrm{p}$ (AMPS/C6) samples in $0.1 \mathrm{M}$ aqueous sodium chloride, ${ }^{5}$ and indicating the existence of two scattering components with fast and slow relaxation times. Most of solutions with $x_{\text {methanol }}=0$ and 0.06 exhibited similar bimodal $A(\tau, k)$.

The mutual diffusion coefficient $D_{\text {fast }}$ of the fast relaxation component was estimated by $D_{\text {fast }}=$ $\lim _{k \rightarrow 0} k^{-2} \sum_{\tau \in \text { fast }} \tau^{-1} A(\tau, k) .{ }^{14}$ Figure $2 b$ shows the results of $D_{\text {fast }}$ for solutions with $x_{\text {methanol }}=0$ and 0.06 , where $k_{\mathrm{B}}$ is the Boltzmann constant, $\eta_{0}$ is the solvent viscosity coefficient, and $k_{\mathrm{B}} T / 6 \pi \eta_{0} D_{\text {fast }}$ corresponds to the apparent hydrodynamic radius. It can be seen that $k_{\mathrm{B}} T / 6 \pi \eta_{0} D_{\text {fast }}$ is a slowly decreasing function of $c$ at $x_{\text {methanol }}=0$, but almost independent of $c$ at $x_{\text {methanol }}=0.06$.

On the other hand, we may estimate (the upper bound of) the weight fraction $w_{\text {slow }}$ of the slow relaxation component in dilute solution using the approximated equation ${ }^{14}$

$$
w_{\text {slow }} M_{\text {slow }} \approx M_{\text {app }} \lim _{k \rightarrow 0}\left[\sum_{\tau \in \text { slow }} A(\tau, k)\right] /\left[\sum_{\tau \in \text { all }} A(\tau, k)\right]
$$

Here $M_{\text {slow }}$ is the molar mass of the slow relaxation component, the lower bound of which may be estimated from the hydrodynamic radius of the slow relaxation component (obtained from $A(\tau, k)$ of the component) in the procedure applied in the previous study. ${ }^{5}$ The upper bound of $w_{\text {slow }}$ obtained by the above method was less than $0.5 \%$ both at $x_{\text {methanol }}=0$ and 0.06 . Therefore, although the slow relaxation component was detectable by light scattering due to its very high scattering power, it may not affect sedimentation equilibrium results. At present, we have no information about the origin of the coexistence of a tiny amount of large aggregtaes.

\section{DISCUSSION}

\section{Theoretical Basis}

In previous publications, ${ }^{5,6}$ we have already demonstrated that $\mathrm{p}$ (AMPS/C6) forms a uni-core micelle in aqueous media if the molecular weight is low enough. We here adopt the same uni-core micelle model for our $\mathrm{p}$ (AMPS/C6) sample; the suitability of the model will be checked in the following.

For star-like micelles, the internal free energy change $\Delta_{m} / m$ (per polymer chain) due to micellization is written as ${ }^{15}$

$$
\Delta_{m} / m=\left(-\delta m+\sigma m^{2}+4 \pi R_{\mathrm{c}}^{2} \gamma\right) / m
$$

where $m$ is the aggregation number, $\delta$ is the free energy gain when a hydrophobic moiety of a polymer chain enters in the micelle core from the solvent, $\sigma$ is the free energy due to interaction between two coronal chains of the micelle, and the third term is the interfacial free energy of the micelle core $\left(R_{\mathrm{c}}\right.$ : the radius of the micellar core; $\gamma$ : the interfacial tension). The optimum aggregation number is equal to $\left(4 \pi R_{\mathrm{c}}{ }^{2} \gamma / \sigma\right)^{1 / 2}$, and in what follows, we consider the monodisperse micellar solution with this optimum aggregation number.

Under the micellization equilibrium, the association constant $K_{m}$ is determined by the equilibrium condition $\mu_{m}=$ $m \mu_{1}$, where $\mu_{m}$ and $\mu_{1}$ are the chemical potentials of $m$-mer and unimer, respectively. Thus, we need chemical potential expressions applicable over a wide polymer concentration range. Here, we use the single-contact approximation to the micellar system, and apply Sato et al.'s expression ${ }^{11}$ of $\mu_{m}$ given by

$$
\frac{\mu_{m}}{k_{\mathrm{B}} T}=\frac{\mu_{m}^{\circ}}{k_{\mathrm{B}} T}+\ln C_{m}+F_{5}^{\prime}(\phi)+m M_{1} \tilde{F}(\phi)
$$

where, $\mu_{m}{ }^{\circ}$ and $C_{m}$ are the standard chemical potential (or the internal free energy) and the molar concentration of $m$-mer, respectively, $M_{1}$ is the molecular weight of unimer, and $F^{\prime}{ }_{5}(\phi)$ and $\tilde{F}(\phi)$ are known functions of the total volume frcation $\phi$ of the polymer; $F^{\prime}{ }_{5}(\phi)$ necessary in what follows is defined by

$$
F_{5}^{\prime} \equiv \frac{5 \phi-3 \phi^{2}}{3(1-\phi)^{2}}-\ln (1-\phi)
$$


(We have neglected an attractive interaction term of the chain end. ${ }^{11}$ ) The chemical potential of unimer $\mu_{1}$ can be calculated by eq 2 with $m=1$. The above expression of $\mu_{m}$ was originally derived on the basis of the scaled particle theory for polydisperse wormlike spherocylinders, and may be a good approximation to polymer systems with lower molar mass and lower degree of branching.

Applying this $\mu_{m}$ to the equilibrium condition, we have the following equation

$$
\begin{aligned}
K_{m} & \equiv \frac{C_{m}}{C_{1}{ }^{m}}=\frac{\left(1-w_{1}\right) / m}{w_{1}{ }^{m}\left(1000 c / M_{1}\right)^{m-1}} \\
& =\exp \left[-\Delta_{m}-(m-1) F_{5}^{\prime}(\phi)\right]
\end{aligned}
$$

Here, $w_{1}$ is the weight fraction in the total polymer, $M_{1}=$ $1.69 \times 10^{4}$ in our system, and $\Delta_{m} \equiv\left(\mu_{m}{ }^{\circ}-m \mu_{1}{ }^{\circ}\right) / k_{\mathrm{B}} T$ is the internal free energy change due to micellization being identical with that given in eq 1 except for a constant term. For given $m$, $\Delta_{m}, c$, and $\phi$, we can calculate $w_{1}$ from the above equation.

Sato et al. ${ }^{11}$ also formulated the apparent molecular weight $M_{\text {app }}$ obtained by sedimentation equilibrium, which is written as

$$
M_{\mathrm{app}}^{-1}=M_{\mathrm{w}}{ }^{-1}+2\left(\Gamma_{2,0}+A_{2, \mathrm{w}}\right) c
$$

where $M_{\mathrm{w}}$ is the weight-average molar mass, $\Gamma_{2,0}$ is the apparent second virial coefficient for the hard-core potential, and $A_{2, \mathrm{w}}$ is the second virial coefficient with respect to the electrostatic and hydrophobic interactions. (The original paper dealt with the quantity $Z(r)$, which can be identified with $M_{\text {app }}{ }^{-1}$ in a good approximation.) The coefficient $\Gamma_{2,0}$ is calculated by

$$
\begin{aligned}
\Gamma_{2,0}= & \frac{\pi d^{2} N_{\mathrm{A}}}{4 M_{\mathrm{L}}}\left[\frac{F_{6}}{d M_{\mathrm{L}}}+\frac{F_{2}}{M_{\mathrm{w}}}+\frac{F_{7}}{M_{\mathrm{n}}}\right. \\
& \left.-\left(\frac{1}{M_{\mathrm{n}}}-\frac{1}{M_{\mathrm{w}}}\right) F_{2}\left(1+\phi F_{2}\right)\right]
\end{aligned}
$$

where $d$ are $M_{\mathrm{L}}$ are the hard-core diameter and the molar mass per unit contour length of the polymer chain, respectively, $M_{\mathrm{n}}$ is the number average molar mass, and $N_{\mathrm{A}}$ is the Avogadro constant. The functions $F_{2}, F_{6}$, and $F_{7}$ are defined by

$$
\begin{aligned}
& F_{2} \equiv \frac{8-7 \phi+3 \phi^{2}}{3(1-\phi)^{3}}, \\
& F_{6} \equiv 2 \frac{1+\phi}{(1-\phi)^{4}}, \\
& F_{7} \equiv \frac{2}{3}\left[\frac{4+\phi+\phi^{2}}{(1-\phi)^{4}}\right]
\end{aligned}
$$

The volume fraction of the total polymer can be calculated by $\phi=\pi d^{2} N_{\mathrm{A}} c / 4 M_{\mathrm{L}}$, while the average molar masses by $M_{\mathrm{w}} / M_{1}=w_{1}+\left(1-w_{1}\right) m$ and $M_{1} / M_{n}=w_{1}+\left(1-w_{1}\right) m^{-1}$. It is noted that the equality between $M_{\text {app }}{ }^{-1}$ and the reciprocal of the osmotic compressibility $(R T)^{-1} \partial \Pi / \partial c$ does not strictly hold for multicomponent polymer solutions.

On the other hand, $A_{2, \mathrm{w}}$ for amphiphilic copolymers may be calculated by

$$
A_{2, \mathrm{w}}=\frac{N_{\mathrm{A}}}{2 \bar{M}_{0}^{2}}\left[(1-x)^{2} \beta_{\mathrm{el}}+x^{2} \beta_{\mathrm{v}}\right]
$$

where $\bar{M}_{0}$ is the average monomer molar mass, $x$ is the content of hydrophobic monomer unit, and $\beta_{\mathrm{el}}$ and $\beta_{\mathrm{v}}$ are the binary cluster integrals with respect to the electrostatic and hydrophobic interactions, respectively. The theory of polyelectrolytes $^{16,17}$ provides us the following equation of $\beta_{\mathrm{el}}$

$$
\beta_{\mathrm{el}}=\frac{2 h^{2}}{\kappa} R(y)
$$

Here, $h$ is the contour length per monomer unit, $\kappa$ is the reciprocal of the Debye screening length, and $y \equiv$ $2 \pi v_{\text {eff }}^{2} Q e^{-\kappa d} / \kappa$ with the effective charge density $v_{\text {eff }}$ and the Bjerrum length $Q$. In this study, $v_{\text {eff }}$ was calculated based on the Philip-Wooding theory ${ }^{18}$ for charged cylinder model. The function $R(y)$ is given in ref 16. Taking into account the contribution of counter ions of the copolymer to the ionic strength, we calculate $\kappa$ by

$$
\kappa^{2}=8 \pi Q N_{\mathrm{A}}\left(\frac{C_{\mathrm{s}}}{1000}+\frac{1-x}{2 \bar{M}_{0}} c\right)
$$

The mutual diffusion coefficient $D_{\text {fast }}$ in micellar solutions at finite concentrations may be written as ${ }^{15,19}$

$$
\frac{k_{\mathrm{B}} T}{6 \pi \eta_{0} D_{\text {fast }}}=\frac{M_{\mathrm{app}}\left(1+k_{\mathrm{H}}^{\prime} c / c^{*}\right)}{M_{\mathrm{w}}(1-\bar{v} c)} R_{\mathrm{H}}
$$

where $\bar{v}$ is the polymer partial specific volume, $k_{\mathrm{H}}^{\prime}$ is the strength of the intermolecular hydrodynamic interaction (corresponding to the Huggins coefficient for the polymer solution viscosity), $c^{*}$ is the overlap concentration, and $R_{\mathrm{H}}$ is the average hydrodynamic radius given by

$$
\frac{R_{\mathrm{H}}}{M_{\mathrm{w}}}=\left[\frac{w_{1} M_{1}}{R_{\mathrm{H}, 1}}+\frac{\left(1-w_{1}\right) m M_{1}}{g_{\mathrm{H}, m} R_{\mathrm{H}, m}(\text { linear })}\right]^{-1}
$$

Here, $R_{\mathrm{H}, 1}$ and $R_{\mathrm{H}, m}$ (linear) are the hydrodynamic radii of the linear chains with the molar masses of $M_{1}$ and $m M_{1}$, respectively, and $g_{\mathrm{H}, m}$ is the $g$-factor with respect to the hydrodynamic radius for the $m$-mer, depending on the branching architecture. The overlap concentration $c^{*}$ may be calculated by ${ }^{15}$

$$
c^{*}=3 M_{\mathrm{w}} / 4 \pi N_{\mathrm{A}} R_{\mathrm{H}}^{3}
$$

\section{Analysis of Experimental Results}

Using the above theoretical expressions, we analyze our experimental $M_{\text {app }}$ and $D_{\text {fast }}$ results for p(AMPS/C6) solutions. To calculate $M_{\text {app }}$ as a function of $c$, we need the following parameters: $m, \Delta_{m}, d, M_{\mathrm{L}}, h, v_{\text {eff }}$, and $\beta_{\mathrm{v}}$. Among them, we have $h=0.25 \mathrm{~nm}$ for vinyl polymers, ${ }^{20}$ and $M_{\mathrm{L}}=\bar{M}_{0} / h=$ $835 \mathrm{~nm}^{-1}$. The effective charge density $v_{\text {eff }}$ can be calculated from the linear charge density $\left[=(1-x) / h=3.4 \mathrm{~nm}^{-1}\right]$ and $d .^{18}$ Thus the remaining unknown parameters are $m, \Delta_{m}, d$, and $\beta_{\mathrm{v}}$.

At $x_{\text {methanol }}=1$ and 0.31 , where the micelle is not formed, $M_{\text {app }}$ is determined only by $d$ and $\beta_{\mathrm{v}}$. If $d$ is calculated from the experimental partial specific volume $\bar{v}$ ( $c f$. Table II) by $d=\left(4 \bar{v} M_{\mathrm{L}} / \pi N_{\mathrm{A}}\right)^{1 / 2}$, we can fit eq 5 to the $M_{\text {app }}$ data at $x_{\text {methanol }}=1$ and 0.31 with $\beta_{\mathrm{v}}=-0.3 \mathrm{~nm}^{3}$. Even at 
Table III. Values of parameters determined from $M_{\text {app }}$ data

\begin{tabular}{ccccc}
\hline$x_{\text {methanol }}$ & $d / \mathrm{nm}$ & $\beta_{v} / \mathrm{nm}^{3}$ & $m$ & $\Delta_{m} / m$ \\
\hline 0 & 1.17 & -0.8 & 4 & -6.87 \\
0.06 & 1.5 & -0.3 & 2 & -4.35 \\
0.13 & 1.5 & -0.3 & 2 & -3.80 \\
$0.31 \& 1$ & 0.96 & -0.3 & - & - \\
\hline
\end{tabular}

$x_{\text {methanol }}=0$ where micelle is formed, $M_{\text {app }}$ in a concentrated region is little dependent on $m$ and $\Delta_{m}$, so that we can similarly determine values of $d$ and $\beta_{\mathrm{v}}$ by fitting high concentration $M_{\text {app }}$ data. Values of $m$ and $\Delta_{m}$ were determined afterward by fitting lower concentration $M_{\text {app }}$ data.

At $x_{\text {methanol }}=0.06$ and 0.13 , we had to choose $d$ values larger than those estimated from $\bar{v}$ to fit eq 5 to high concentration data of $M_{\text {app}}$. This may have something to do with the solvation of methanol to the $\mathrm{p}$ (AMPS/C6) chain. Here, we assumed that $\beta_{\mathrm{v}}$ at $x_{\text {methanol }}=0.06$ and 0.13 is equal to that at $x_{\text {methanol }}=1$ and 0.31 , and determined $d$ to fit eq 5 to $M_{\text {app }}$ data at high $c$. Values of $m$ and $\Delta_{m}$ were determined from $M_{\text {app }}$ data at low $c$.

As shown by solid curves in Figure 1, we can satisfactorily fit the theoretical curve to experimental data at every $x_{\text {methanol }}$. Values of $d, \beta_{\mathrm{v}}, m$, and $\Delta_{m} / m$ used in the fittings are listed in Table III. The aggregation number $m$ is 4 at $x_{\text {methanol }}=0$, but reduces to 2 at $x_{\text {methanol }}=0.06$ and 0.13 , and micellization does not take place at $x_{\text {methanol }}>0.3$. Furthermore, $\Delta_{m} / m$ (the free energy change per polymer chain due to micellization) increases with increasing $x_{\text {methanol }}$. Destabilization of the micelle by addition of methanol arises from diminishing the hydrophobic interaction. From the same reason, we can expect an increase of the repulsion among coronal chains and then of $\sigma$ (the interaction free energy between two coronal chains) in eq 1 as $x_{\text {methanol }}$ increases, which may lead the reduction of the optimum aggregation number $\left[=\left(4 \pi R_{\mathrm{c}}{ }^{2} \gamma / \sigma\right)^{1 / 2}\right]$.

The diffusion coefficient $D_{\text {fast }}$ for the micellar solution is calculated by eqs $11-13$, which include $R_{\mathrm{H}, 1}, R_{\mathrm{H}, m}$ (linear), $g_{\mathrm{H}, m}$, and $k_{\mathrm{H}}^{\prime}$. In general, the hydrodynamic radius $R_{\mathrm{H}}$ of linear polymers has a power-law dependence on the degree of polymerization $N_{0}: R_{\mathrm{H}}=K^{\prime}{ }_{\mathrm{H}} N_{0}{ }^{a_{\mathrm{H}}}$ with two parameters $K_{\mathrm{H}}^{\prime}$ and $a_{\mathrm{H}}$ characteristic to the polymer-solvent system. The $N_{0}$ dependence of $R_{\mathrm{H}}$ of $\mathrm{p}(\mathrm{AMPS} / \mathrm{C} 6)$ with $x=0.15$ at $x_{\text {methanol }}=$ 0 and 0.06 should be similar to that for the AMPS homopolymer in aqueous solution, ${ }^{5}$ but due to the hydrophobic interaction among hexyl groups within the chain the solvent quality may be more or less reduced. We have used $K_{\mathrm{H}}^{\prime}$ $(=0.22 \mathrm{~nm})$ and $a_{\mathrm{H}}(=0.58)$ values of the AMPS homopolymers in $0.5 \mathrm{M}$ aqueous $\mathrm{NaCl}$ which may be a slightly poorer solvent than $0.2 \mathrm{M}$ aqueous $\mathrm{LiClO}_{4}{ }^{21}$

If hydrophobes near one of $\mathrm{p}(\mathrm{AMPS} / \mathrm{C} 6)$ chain ends are incorporated into the core, our uni-core micelle of the $m$-mer can be regarded as an $m$-arm star polymer. On the other hand, when hydrophobes in the middle portion of the chain are included into the core, the uni-core micelle should be a $2 m$-arm star polymer. It is known that the $g$-factor of the $f$-arm star polymer with respect to the hydrodynamic radius is given by ${ }^{22}$

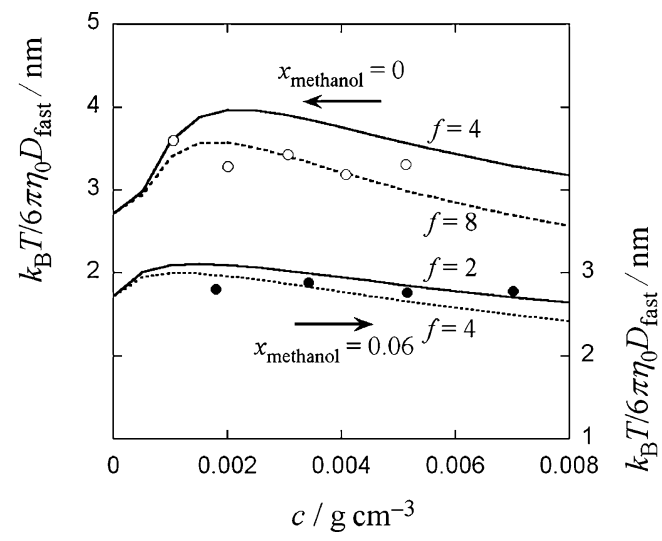

Figure 3. Comparison of experimental data of the diffusion coefficient for $\mathrm{p}$ (AMPS/C6) micelles at $x_{\text {methanol }}=0$ and 0.06 with the theory for the $f$-arm star-like micelle.

$$
g_{\mathrm{H}, m}=\frac{f^{1 / 4}}{[2-f+\sqrt{2}(f-1)]^{1 / 2}} \frac{1-0.068-0.0075(f-1)}{1-0.068}
$$

Finally, linear flexible homopolymers in good solvents take values of 4-7 for the strength $k_{\mathrm{H}}^{\prime}$ of the intermolecular hydrodynamic interaction. ${ }^{15}$ Choosing $k_{\mathrm{H}}^{\prime}=8$ which is slightly larger than those for linear polymers, we obtain solid and dashed curves in Figure 3. Here, the solid and dashed curves correspond to theoretical values for $f=m$ and $2 m$, respectively. Experimental data points at both $x_{\text {methanol }}=0$ and 0.06 are between the solid and dashed curves. This demonstrates that the micelle of $\mathrm{p}(\mathrm{AMPS} / \mathrm{C} 6)$ is star-like and its hydrophobic core consists of hexyl groups attaching to random positions of the copolymer chain.

\section{Comparison with the Corresponding Low-Molar Mass Amphilile}

Recently, Ruso et $a l .{ }^{23}$ investigated the micellization behavior of $n$-hexyltrimethylammonium bromide (C6TAB) in aqueous $0.2-0.7 \mathrm{M} \mathrm{NaBr}$, of which hydrophobic moiety is the same as $\mathrm{p}(\mathrm{AMPS} / \mathrm{C} 6)$. The aggregation number reported for C6TAB (3-4) is close to our aggregation number of $\mathrm{p}$ (AMPS/C6) chains in aqueous $\mathrm{LiClO}_{4}$, but C6TAB forms the micelle only at high concentrations $(0.4-0.6 \mathrm{M}$ of C6TAB). For $\mathrm{p}$ (AMPS/C6), the micelle is formed at concentration as low as $0.002 \mathrm{~g} / \mathrm{cm}^{3}$, which corresponds to $10^{-3} \mathrm{M}$ of the hexyl group. The remarkable stabilization of the $\mathrm{p}(\mathrm{AMPS} / \mathrm{C} 6)$ micelle may be owing to (1) hexyl groups are concentrated within the copolymer chain domain and (2) the charged sulphate group is apart from the hexyl group. Although the aggregation number of $\mathrm{p}$ (AMPS/C6) chains is four, the core of the copolymer micelle may consist of hexyl groups more than four, which can stabilize the copolymer micelle more.

Since the p(AMPS/C6) micelle is star-like, not all hexyl groups attaching to the copolymer chain are incorporated into the hydrophobic core. The high critical aggregation concentration for C6TAB indicates that hydrophobicity of the hexyl group is not so strong that the hexyl group is completely helped exposing to aqueous medium. 


\section{CONCLUSION}

We have investigated the micellization equilibrium of the statistical copolymer p(AMPS/C6) in water, methanol, and their mixtures. In aqueous $0.2 \mathrm{M} \mathrm{LiClO}_{4}, \mathrm{p}(\mathrm{AMPS} / \mathrm{C} 6)$ forms a uni-core star-like tetramer, and both stability and aggregation number of the micelle sharply decrease by addition of methanol which reduces the hydrophobic interaction among hexyl groups attaching to copolymer chains. The micellization equilibrium and also micellar conformation were successfully described by the proposed theory.

Received: September 20, 2008 Accepted: November 20, 2008 Published: January 15, 2009

\section{REFERENCES}

1. A. Hashidzume, Y. Morishima, and K. Szczubialka, in "Handbook of Polyelectrolytes and Their Applications," S. K. Tipathy, J. Kumar, and H. Nalwa, Ed., American Scientific Publishers, Stevenson Ranch, CA, 2002, vol. 2, pp 1-63.

2. "Hydrophilic Polymers. Performance with Environmental Acceptability,” J. E. Glass, Ed., American Chemical Society, Washington, DC, 1996.

3. "Associative Polymers in Aqueous Solutions," J. E. Glass, Ed., American Chemical Society, Washington, DC, 2000.

4. "Stimuli-Responsive Water Soluble and Amphiphilic Polymers," C. L. McCormick, Ed., American Chemical Society, Washington, DC, 2001.
5. A. Hashidzume, A. Kawaguchi, A. Tagawa, K. Hyoda, and T. Sato, Macromolecules, 39, 1135 (2006).

6. R. Nojima, A. Hashidzume, and T. Sato, Macromol. Symp., 249-250, 502 (2007).

7. E. Sprong, D. De Wet-Roos, M. P. Tonge, and R. D. Sanderson, J. Polym. Sci., Part A: Polym. Chem., 41, 223 (2003).

8. J. Chiefari, Y. K. Chong, F. Ercole, J. Krstina, J. Jeffery, T. P. T. Le, R. T. A. Mayadunne, G. F. Meijs, C. L. Moad, G. Moad, E. Rizzardo, and S. H. Thang, Macromolecules, 31, 5559 (1998).

9. Y. Mitsukami, M. S. Donovan, A. B. Lowe, and C. L. McCormick, Macromolecules, 34, 2248 (2001).

10. H. Fujita, "Foundation of Ultracentrifugal Analysis," Wiley-Interscience, New York, 1975.

11. T. Sato, Y. Jinbo, and A. Teramoto, Macromolecules, 30, 590 (1997).

12. T. Kawata, A. Hashidzume, and T. Sato, Macromolecules, 40, 1174 (2007).

13. L. Wu, T. Sato, H.-Z. Tang, and M. Fujiki, Macromolecules, 37, 6183 (2004).

14. M. Kanao, Y. Matsuda, and T. Sato, Macromolecules, 36, 2093 (2003).

15. Y. Matsuda, R. Nojima, T. Sato, and H. Watanabe, Macromolecules, 40, 1631 (2007).

16. M. Fixman and J. Skolnick, Macromolecules, 11, 863 (1978).

17. K. Kawakami and T. Norisuye, Macromolecules, 24, 4898 (1991).

18. J. R. Philip and R. A. Wooding, J. Chem. Phys., 52, 953 (1970).

19. T. Kanematsu, T. Sato, Y. Imai, K. Ute, and T. Kitayama, Polym. J., 37, 65 (2005).

20. Y. Matsuda, Y. Miyazaki, S. Sugihara, S. Aoshima, K. Saito, and T. Sato, J. Polym. Sci., Part B: Polym. Phys., 43, 2937 (2005).

21. L. W. Fisher, A. R. Sochor, and J. S. Tan, Macromolecules, 10, 949 (1977).

22. J. F. Douglas, J. Roovers, and K. Freed, Macromolecules, 23, 4168 (1990).

23. J. M. Ruso, D. Attwood, P. Taboada, and V. Mosquera, Colloid Polym. Sci., 280, 336 (2002). 